\title{
False chordae tendineae in right ventricle of adult human hearts - morphological aspects
}

Adam Kosiński ${ }^{1}$, Marek Grzybiak ${ }^{1}$, Ada Dubaniewicz ${ }^{1}$, Krzysztof Kędziora², Wojciech Makarewicz ${ }^{3}$, Dariusz Kozłowski ${ }^{4}$

1Department of Clinical Anatomy, Medical University of Gdansk, Poland

2Pneumonology Department, Medical University of Gdansk, Poland

${ }^{3}$ Department of Oncological Surgery, Medical University of Gdansk, Poland

4 Department of Cardiology and Electrotherapy, Medical University of Gdansk, Poland

Submitted: 16 September 2011

Accepted: 11 March 2012

Arch Med Sci 2012; 8, 5: 834-840

DOI: 10.5114/aoms.2012.31617

Copyright (C) 2012 Termedia \& Banach

\section{Abstract}

Introduction: False chordae tendineae are fibrous-muscular bundles which do not interconnect with right atrioventricular valves. The structures have occasionally been described in the right ventricle. There are reports suggesting their influence on electromechanical processes taking place in the heart, in thromboembolic events as well as in the course of cardiac invasive procedures. The objective of the study was to perform a macroscopic evaluation of false chordae tendineae in the right ventricle.

Material and methods: The research specimens consisted of 100 hearts of adult humans, aged from 18 to 59 years, fixed in a solution of $10 \%$ formaldehyde and $98 \%$ ethanol. The ratio of false chordae tendineae to individual elements of the right ventricle, such as its walls, papillary muscles, septomarginal trabecula and the apex of the ventricle, was examined.

Results: During examination, six types of chordae tendineae were described based on the criterion of the type of structures they connected. The most common were false chordae connecting ventricle walls within its apex, while the least common were individual segments of papillary muscles. The research proved that the examined structures are morphologically extremely diverse. Substantial clinical implications of their presence seem very probable.

Conclusions: The present work is the first of a scheduled series devoted to the problem of false chordae tendineae. Further analyses will cover the subject of morphological aspects in a microscopic perspective.

Key words: false chordae tendineae, right ventricle, heart.

\section{Introduction}

False chordae tendineae (FChT) are described in the literature as fibrousmuscular bundles present in ventricles, not interconnecting, unlike "true" chordae, with atrioventricular valves. Until now, there has been more interest in presence of FChT in the left ventricle. Undoubtedly, they are more visible and, due to their bigger size, their role has been described as mechanical - protecting the ventricle from excessive dilation $[1,2]$. In the literature there are numerous reports suggesting significant influence of the structures on electromechanical processes in the heart. There have been papers reporting a possible arrhythmogenic role of FChT, as well as diagnostic errors in echocardiography arising mostly from false valuation

\author{
Corresponding author: \\ Prof. Dariusz Kozłowski \\ Department of Cardiology \\ and Electrotherapy \\ Medical University of Gdansk \\ 7 Debinki St \\ 80-211 Gdansk, Poland \\ Phone: +48 583493910 \\ E-mail: dkozl@gumed.edu.pl
}


of those elements [3-5]. The role of FChT has also been noticed in the aspect of pulmonary thromboembolism, although presented analyses referred to the left ventricle [6]. The influence of the described structures on the procedure of implantation of the cardiac pacemaker's electrode also seems remarkably important [7].

Despite the clinical problem arising from the chordae, they still have not found a proper place in anatomy reference books, and existing compilations treat them very cursorily.

Still, the problem of morphology of FChT in the right ventricle along with substantial clinical implications of their presence justifies the need to undertake a detailed analysis of the structures.

\section{Material and methods}

Material for the research consisted of the hearts of 100 human adults of both sexes, aged from 18 to 59 years, without visible pathological lesions or malformations, deceased due to extracardiac reasons. All hearts were fixed in a solution of $10 \%$ formaldehyde and $98 \%$ ethanol.

The right ventricle was opened with an incision running $0.5-1 \mathrm{~cm}$ to the back from the sharp edge, continuing it towards the outlet of the superior vena cava, and down towards the apex of the ventricle. Annulus fibrosus of the tricuspid valve was cut in the area of the commissures of anterior and posterior leaflets. Then, the inside of the right ventricle was examined in terms of existing FChT, paying attention to their number, degree of formation, topography, location of attachments as well as length and thickness. The ratio of FChT to individual elements of the right ventricle, such as its walls, papillary muscles, septomarginal trabecula and the apex of the ventricle, was examined (Figure 1). To improve the clarity of the description, the angles between the walls of the ventricle were included. On the basis of the chordae's location and their course, a division into individual types was proposed, based on a formerly determined classifica-

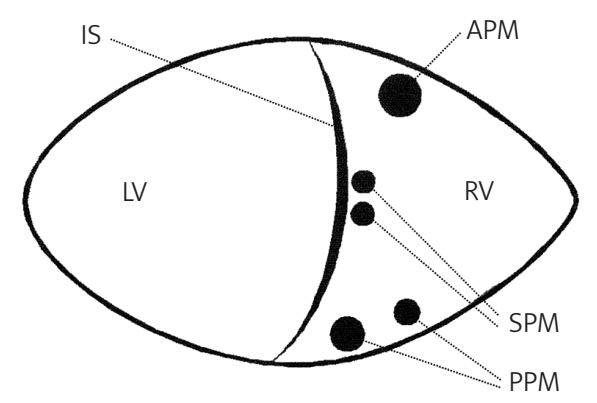

Figure 1. A scheme of heart cross-section at the level of the ventricles

$L V$ - left ventricle, $R V$ - right ventricle, IS - interventricular septum, APM - anterior papillary muscle, SPM - septal papillary muscles, PPM - posterior papillary muscles tion according to Grzybiak and Bobiński [8] and referring to FChT in the left ventricle. A selected part of the material was statistically evaluated with the $\chi^{2}$ test.

\section{Results}

False chordae tendineae in the right ventricle were observed in all hearts; they were divided into groups according to their location and connection of the individual structures of the ventricle. In the study, as stated before, a distinction according to Grzybiak and Bobiński [8] was used for FChT in the left ventricle, taking into account morphological variations of the right ventricle. Six types of chordae were discriminated, connecting:

I - papillary muscles with the outer wall of the ventricle,

II - individual segments of the papillary muscles,

III - papillary muscles with the interventricular septum,

IV - various papillary muscles,

$\mathrm{V}$ - the walls of the ventricle around its apex,

$\mathrm{VI}$ - septomarginal trabecula with other structures of the right ventricle.
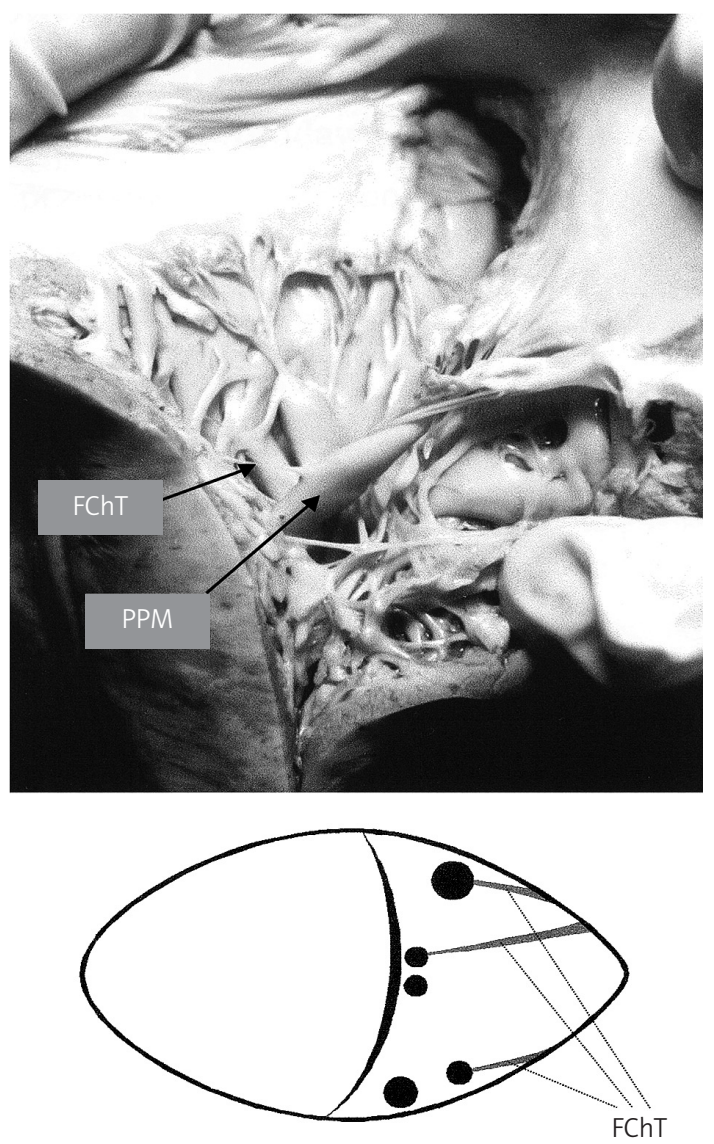

Figure 2. Type I false chordae tendineae (male, 43 years). Scheme layout as in Figure 1 FChT - false chordae tendineae, PPM - posterior papillary muscle 
Chordae connecting anterior, posterior and septal papillary muscles with the anterior or posterior wall of the right ventricle were qualified as type I (Figure 2). The chordae were observed in more than a half of the examined hearts and the average frequency of their appearance was 19\% (Figure 3). They were relatively plural structures. Chordae connecting the posterior papillary muscle with the posterior wall of the right ventricle were the most common. They mostly run from the central part of the muscle and, in case the muscle was multisegmental, usually from its biggest, wall-located part. Chordae connecting anterior papillary muscle with the anterior wall of the right ventricle in most cases appeared individually. Their initial attachment was located at different heights of the muscle. The least common were chordae running from the septal muscles.

The chordae of type I were rather short, less than $1 \mathrm{~cm}$ long, and they ran perpendicularly to the long axis of the muscle.

The chordae connecting individual segments of papillary muscles were qualified as type II (Figure 4). As a rule, this did not refer to the anterior papillary muscle. It is the kind of chordae which occurs the least frequently. In the examined material, they were observed in every third heart, with relative frequency of 5.6\% (Figure 3). They occurred individually or doubly. Most chordae of type II referred to the posterior papillary muscle, which is known to be morphologically very varied. The described chordae always reached its most extended part. Their attachments were mostly located in the middle of the height of the muscle, and when appearing doubly, the other chorda was usually located at the base. Structures connecting septal muscles appeared in a few cases. The chordae of type II were very short, with a length of less than half a centimetre, thin, with a quite well visible arcuate attachment blending in the structure of the papillary muscle.

Type III includes chordae connecting the interventricular septum with papillary muscles (Figure 5). Like type I, they were encountered in over half

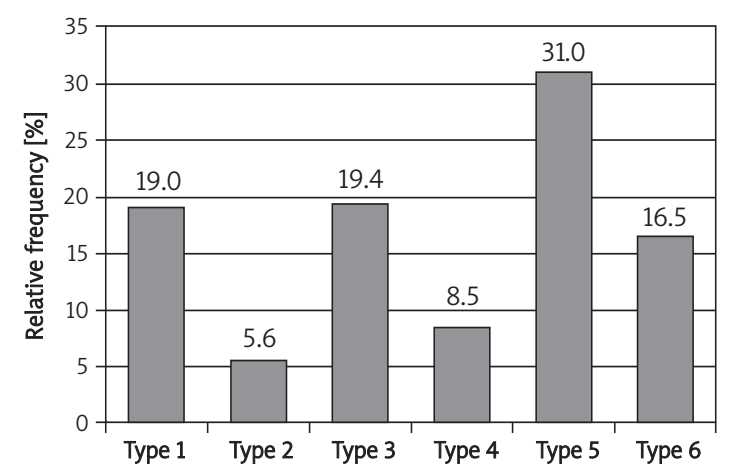

Figure 3. Prevalence of different types of false chordae tendineae of the examined hearts, and their relative frequency was $19.4 \%$ (Figure 3). In many cases, they were plural structures. They originated from the trabecular part of the interventricular septum. The chordae of type III in most cases reached the posterior papillary muscle, as a rule its best developed part. In the examined material, chordae reaching the anterior papillary muscle were also present - from the front part of the trabecular interventricular septum, in most cases in the vicinity of the septomarginal trabecula. Both locations were characteristic for similar morphology of the chordae: they were thin, of average length, with clear tendinous structure, which in the preserved material made them similar to chordae tendineae of the valve apparatus.

Type IV included chordae connecting individual papillary muscles (Figure 6). They were present in slightly more than a third of the examined hearts, with relative frequency of $8.5 \%$ (Figure 3). Most chordae of that type connected the anterior and posterior papillary muscles. The structures were long and quite thick, as for the right ventricle. Usually, they became damaged during routine opening of the cardiac cavity, since they ran through its

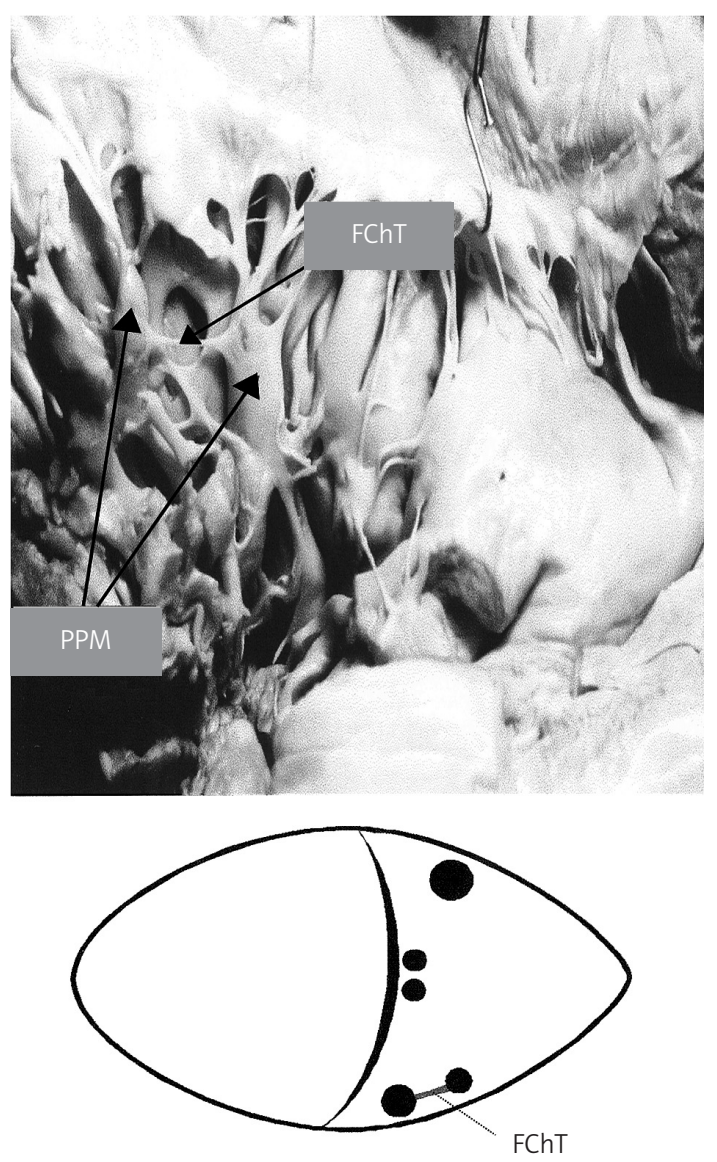

Figure 4. Type II false chordae tendineae (male, 27 years). Scheme layout as in Figure 1 FChT - false chordae tendineae, PPM - posterior papillary muscles 


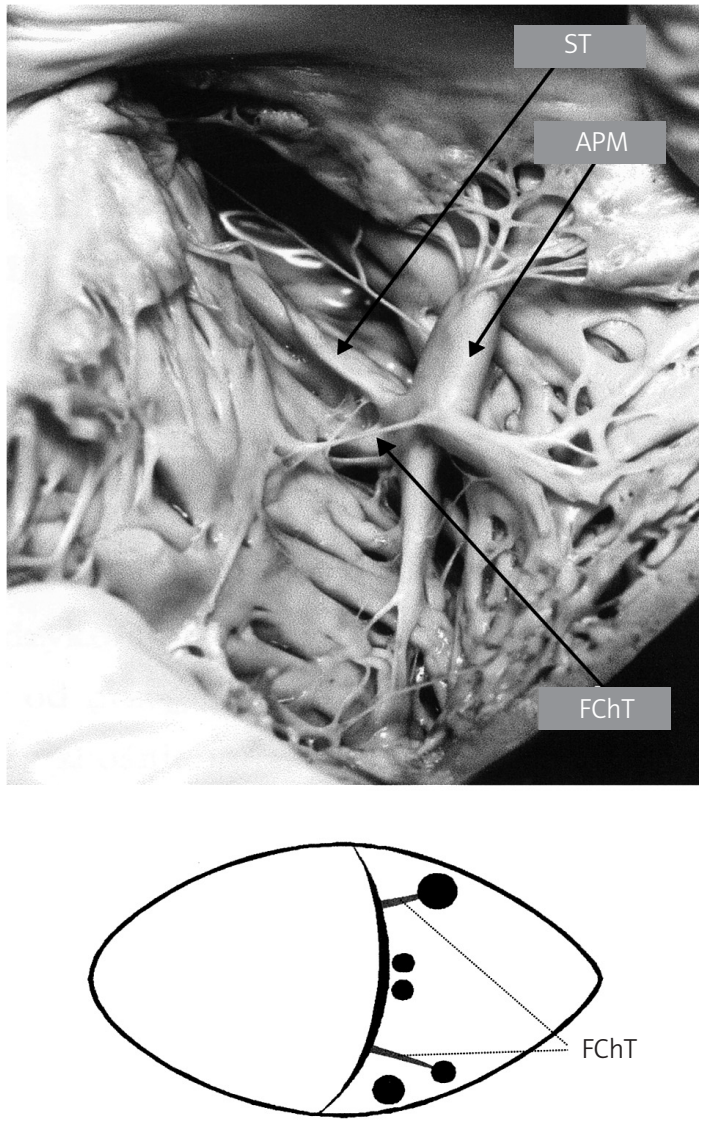

Figure 5. Type III false chordae tendineae (male, 21 years). Scheme layout as in Figure 1

FChT - false chordae tendineae, APM - anterior papillary muscle, ST - septomarginal trabecula

lumen in the upper part. In most cases they connected muscles in their upper or central parts. Less frequent chordae connecting interventricular and posterior muscles were usually shorter and thinner.

Chordae having attachments below papillary muscles were classified as type $\mathrm{V}$ (Figure 7) and most often appeared around the apex of the ventricle. They were the most frequent (present in over 70\% of examined hearts), and their relative frequency amounted to 31\% (Figure 3), often as plural structures. Chordae of type $V$ connected trabeculae carneae present below the bases of papillary muscles. Their morphology was profoundly varied - both long, thick chordae running through the lumen of the ventricle and connecting opposite walls, as well as short, thin ones located more at the walls.

Type VI included chordae connected with the septomarginal trabecula (Figure 8). They were observed in over half of the examined hearts, and their relative frequency was $16.5 \%$ (Figure 3 ). In most cases they were structures connecting the bottom part of the septomarginal trabecula with the interventricular septum. In such cases, usually two (or three) thin, short chordae were observed running parallel to each other.

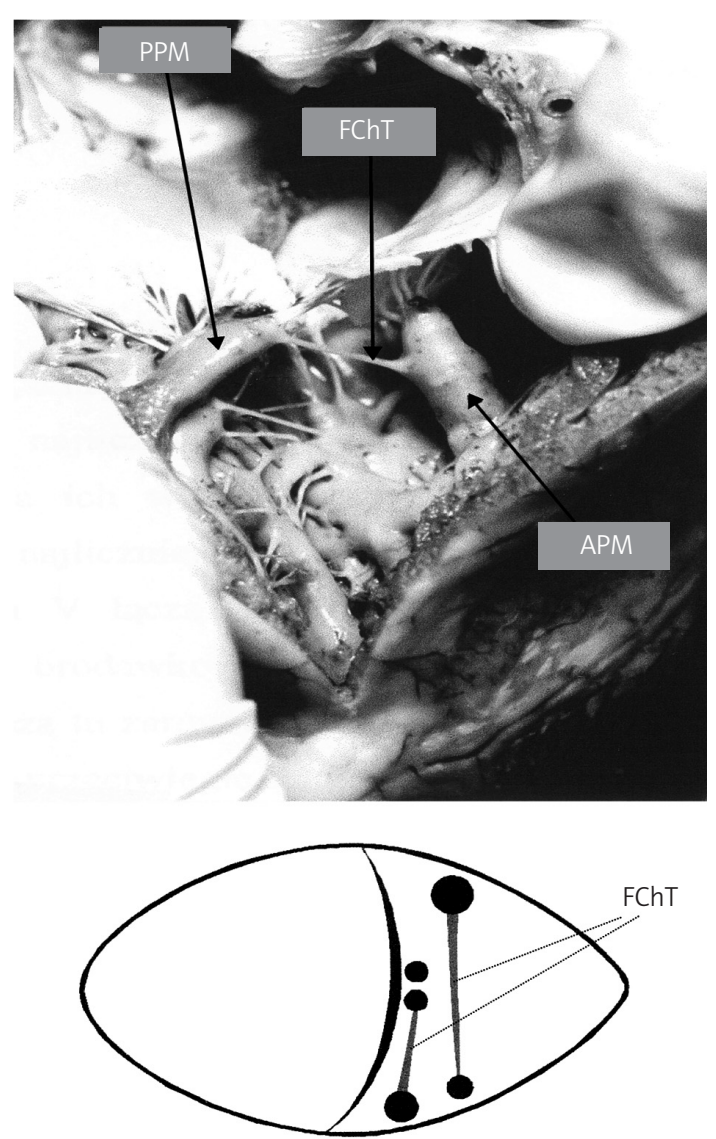

Figure 6. Type IV false chordae tendineae (male, 33 years). Scheme layout as in Figure 1

FChT - false chordae tendineae, APM - anterior papillary muscle, PPM - posterior papillary muscle

The correlation between the type of FChT and factors such as sex or age was not evaluated in statistical analysis.

\section{Discussion}

False chordae tendineae in the right ventricle of a human heart have been studied very cursorily so far. In the available literature there are no case studies referring to the build, localization and frequency of occurrence of the structures. They have rarely been described in the heart during additional examinations, for example echocardiography or when performing a routine autopsy.

The presence of FChT was observed in all examined hearts. The variety of frequency of their occurrence within individual types is very wide. In the right ventricle, mostly represented were FChT of type $\mathrm{V}$, whose attachments are located below the bases of papillary muscles (except chordae reaching the septomarginal trabecula). Due to changeable localization of papillary muscles in the right ventricle, the area of occurrence of FChT of type $V$ varies within the examined population and includes $1 / 3$, even up to $1 / 2$ of the bottom part 

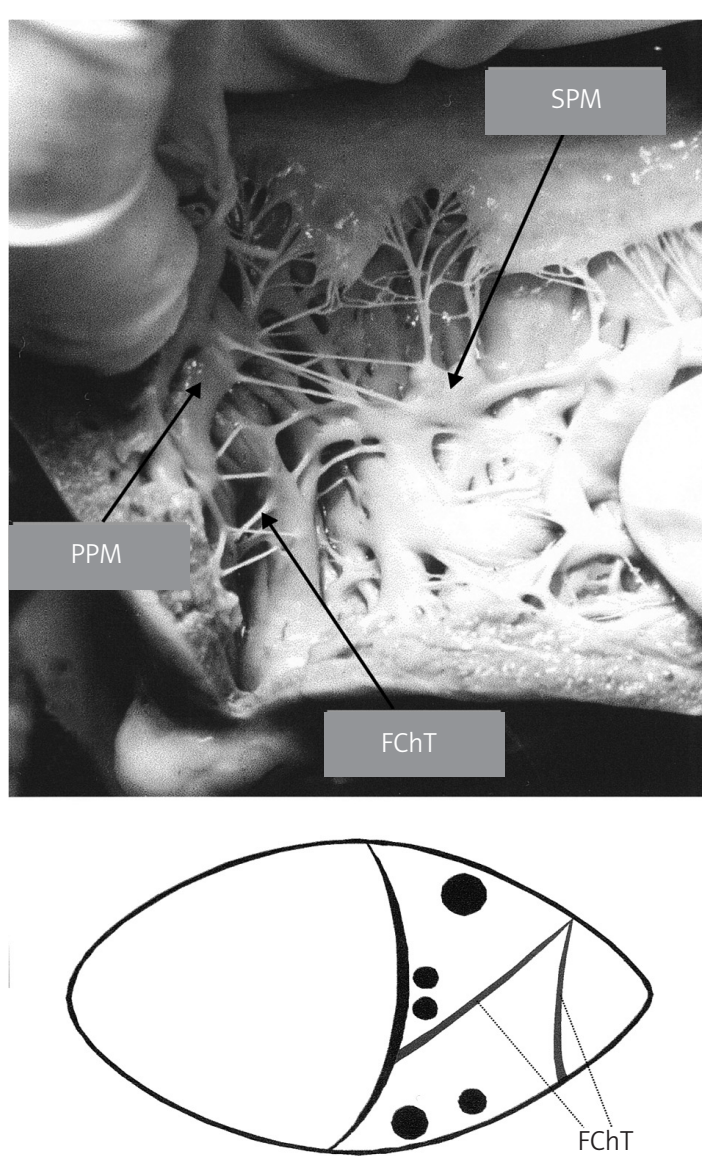

Figure 7. Type $V$ false chordae tendineae (female, 19 years). Scheme layout as in Figure 1

FChT - false chordae tendineae, SPM - septal papillary muscles, PPM - posterior papillary muscle

of the ventricle. Topographically, chordae of that type occupy the largest area in the right ventricle. They also show the greatest morphological diversity. The remaining types of chordae occur in the examined population less frequently and show far less diversity of structure.

Very similar, average frequency of occurrence was observed within two other types of FChT I and III (connection between papillary muscles and respectively outer wall of the ventricle and interventricular septum). No significant correlation between occurrence of the chordae was determined. However, they possess many common characteristics; usually they reach the posterior papillary muscle, are of medium length and thickness and most possess a simple attachment.

Similar to the two previous types, slightly lower average frequency of occurrence was observed in type VI. They are the chordae reaching the septomarginal trabecula. Each of the above types was observed in around a half of examined hearts.

The remaining kinds (type II and IV) occurred far less frequently, connecting segments of papillary muscle and papillary muscles with each other.
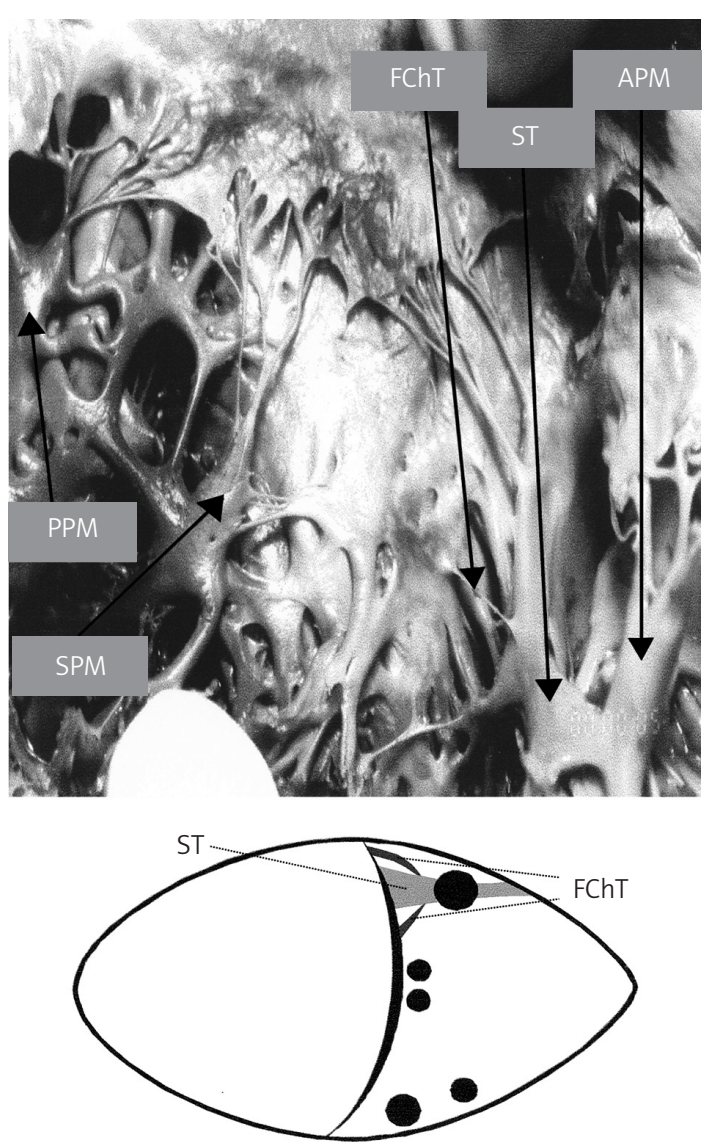

Figure 8. Type VI false chordae tendineae (female, 31 years). Scheme layout as in Figure 1

FChT - false chordae tendineae, APM - anterior papillary muscle, SPM - septal papillary muscles, PPM - posterior papillary muscle, ST - septomarginal trabecula

Turner [1, 2, 9], in his first studies on FChT in the left ventricle of a human heart, used the term "moderator band". This is an obvious analogy to the septomarginal trabecula present in the right ventricle. The expression "moderator band" is also commonly used in literature referring to animals (mammals, e.g. ungulates, carnivores), which do not possess a typical septomarginal trabecula, although multiple muscle bundles running through the lumen of the ventricles are present. Topographically they correspond with FChT occurring in primates; however, their microscopic build is different as they are mostly composed of Purkinje fibres.

The term "false chordae tendineae" is used in medical literature. Clearly, the expression became popular on the basis of macroscopic studies. False chordae tendineae, especially in preserved material, look identical to the chordae tendineae of valve apparatus, visibly different from neighbouring muscle structures. Also, in non-preserved material, differences between chordae are not serious. They are similar, filiform structures, differing only, and often very relatively, in colour. This results from filming 
muscle tissue through the endocardium, which lends false chordae a beige-rose hue. In preserved material, where the muscular tissue changes colour to light brown, the hue differences disappear.

False chordae tendineae in the human heart were described as a clinical problem in reference to the left ventricle. Already in the first publications, they were mentioned as a cause of abnormal acoustic phenomena occurring in the heart. Browicz, paying attention to FChT located in the outflow tract from the left ventricle, noted that by causing the vibrations of flowing blood stream, they may be the reason for unusual sounds [10]. This quite cautious statement draws attention to the connection between turbulent flow and occurring murmurs. At present it is known that in adult humans, FChT without organic disease should not cause flow disorder resulting in occurrence of murmurs.

Along with improving non-invasive cardiac examination, especially echocardiography, there appeared a possibility of precise evaluation of inner heart structure in a living human. In the nineteen seventies and eighties, case studies describing FChT in the left ventricle appeared. These authors paid attention to those structures as a potential source of misdiagnosis when evaluating, for example, a clot located at the wall of the ventricle. The chordae were also discussed as a source of cardiac arrhythmia [3-5], their role was taken into account in preventing the ventricle from dilatating $[1,2]$ or they were not connected with any pathology [11].

False chordae tendineae were mentioned among the causes of functional murmurs observed in children and young adults. Attempts to explain the phenomenon have been present in the literature for many years $[4,12]$. Due to the lack of direct correlation between occurrence of FChT and, according to many authors, observing changeable functional murmurs, connecting both phenomena seems to be very doubtful $[13,14]$. The chordae located in the right ventricle are not a very probable substrate of additional acoustic phenomena, due to the haemodynamic conditions present in its lumen.

In the context of the human cardiac conduction system, the subject of FChT is rarely described in the literature. The right branch of the His bundle runs subendocardially in the front part of the septa and, according to many authors, it invades the septomarginal trabecula, going towards the base of anterior papillary muscle $[15,16]$. Histological examinations of the septomarginal trabecula confirm the presence of the right branch only in a proportion of examined hearts $[17,18]$.

The significance of FChT was also noticed in the aspect of pulmonary thromboembolic problems. The conducted studies pay attention to the possibility of more frequent occurrence of thromboem- bolic incidents in the central nervous system in people who were diagnosed with FChT in the left ventricle during echocardiography [6]. Due to haemodynamic conditions, forming new thromboembolic material in the right ventricle seems to be easier than in the left one. Recurring incidents of pulmonary thromboembolism are known from the literature in spite of such radical methods of treatment as using a filter in the inferior vena cava [19]. With no other risk factors, formation of thromboembolic material in the right ventricle "cut" with FChT seems to be very probable.

Another problem deserving a more thorough discussion is the influence of FChT on introducing and final location of the electrode for heart stimulation. A number of factors seem to determine the process, as well as further intensification of morphological changes [7]. Passing the electrode through the right atrioventricular orifice without a contact with the structures of the valve apparatus gives a greater chance for placing the tip in the apex of the right ventricle. In the examined material it is apparent that quite a clear influence may arise from the shape of anatomical structures located in the bottom part of the right ventricle. Apart from the obstacle caused by the septomarginal trabecula, one should also expect a significant proportion of FChT. Numerous structures around the apex of the ventricle (type $V$ ) and those reaching the septomarginal trabecula (type $\mathrm{VI}$ ) may intensely hamper introduction of the electrode.

False chordae tendineae have also been described in animals [20-24]. The internal structure of the human right ventricle and many primates is similar. The differences refer mostly to the development of the trabeculae carneae and the shape of the papillary muscles. Still, the structures are variable also within the above-mentioned groups [25].

In other mammals, for example in carnivores, a typical septomarginal trabecula does not exist. However, there occur numerous and well-developed FChT. They are very visible while sectioning the heart, especially at almost smooth walls of the right ventricle, since trabeculae carneae of those animals are poorly diversified. Some ungulates, in which the septomarginal trabecula appears, also have a developed system of FChT.

Thorough discussion of FChT in the right ventricle of a human heart, taking into account anatomy, histology and functions, seems to be right and justified. Conducted analyses showed that they constitute permanent elements, creating, along with other structures of the right ventricle, a morphologically uniform build. The presented studies show that the still fragmentary knowledge needs to be deepened during further observations. It seems probable that in the future they will allow a firm definition of the role of FChT 
in the right ventricle and their presumptive connection with heart pathology.

The present work is the first of a scheduled series devoted to the problem of FChT. Further analyses will cover the subject of morphological aspects from a microscopic perspective.

\section{References}

1. Turner W. A human heart with moderate bands in the left ventricle. J Anat Physiol 1893; 27: 19-20.

2. Turner W. Another heart with moderator band in the left ventricle. J Anat Physiol 1896; 30: 568-9.

3. Keren A, Billingham M, Popp RL. Echocardiografic recognition and implications of ventricular hypertrophic trabeculations and aberrant bands. Circulation 1984; 70: 836-42.

4. Perry LW, Ruckman RN, Shapiro SR, Kuehl KS, Galioto FM, Scott LP. Left ventricular false tendons in children: Prevalence as detected by 2-dimensional echocardiography and clinical significance. Am J Cardiol 1983; 52: 1264-6.

5. Suwa M, Hirota $Y$, Nagao H, Kino M, Kawamura K. Incidence of coexistence of left ventricular false tendons and premature ventricular contractions in apparently healthy subjects. Circulation 1984; 70: 793-8.

6. Traboulsi M, Thompson CR, MacDonald RP, Belenkie I, Smith ER. Do left ventricular false tendons provide a cardiac source for cerebrovascural emboli? Circulation 1985; 72: 133.

7. Kozłowski D, Dubaniewicz A, Koźluk E, et al. Morfologiczne aspekty jednojamowej prawokomorowej stałej stymulacji serca. Część I: Położenie elektrody w prawej komorze serca. Elektrofizjologia i Stymulacja Serca 1998; 5: 38-44.

8. Grzybiak M, Bobiński M. Types of chordae tendineae spuriae in left ventricle in the human adult heart. Folia Morphol (Warsz.) 1990; 49: 251-9.

9. Turner W. Moderator band in the left ventricle and tricuspid left auriculo-ventricular valve. J Anat Physiol 1898; 32: 372-6.

10. Browicz T. Über anomale Sehnenfaden im Herzen und deren eventuelle Bedeutung. Virchows Arch Pathol Anat Physiol Klin Med 1896; 145: 649-52.

11. Vered Z, Meltzer RS, Benjamin P, Motro M, Neufild HN. Prevalence and significance of false tendons in the left ventricle as determined by echocardiography. Am J Cardiol 1984; 53: 330-2.

12. Fogel DH. The innocent systolic murmur in children: a clinical study of its incidence and characteristics. Am Heart J 1960; 59: 844-55.

13. Malouf J, Gharzuddine W, Kutayli F. A reappraisal of the prevalence and clinical importance of left ventricular false tendons in children and adults. Br Heart J 1986; 55: 587-91.

14. Wessel A, Bayer C, Pulss W, Kempmann U, Heitzen PH. False chordae tendineae in the left ventricle. Echo- and phonocardiografic findings. Zeitsch Kardiol 1985; 74: 303-7.

15. Podlecki K. Morphology of the anterior papilary muscle in the right ventricle in human heart. Folia Morphol (Warsz.) 1987; 46: 187-97.

16. Tawara $\mathrm{S}$. The conduction system of the mammalian heart. Imperial College Press, London 2000.

17. Bojsen-Moller F, Tranum-Jensen J. On nerves and nerve endings in the conducting system of the moderator band (septomarginal trabecula). J Anat 1971; 108: 387-95.
18. Truex RC, Copenhaver WM. Histology of the moderator band in man and other mammals with special reference to the conduction system. Am J Anat 1947; 80: 173-202.

19. Braunwald E. Heart disease. W.B. Saunders, Philadelphia 1992.

20. Abramson D, Margolin MA. A Purkinje conduction network in the myocardium of the mammalian ventricles. J Anat 1935; 70: 250-9.

21. Armiger LC, Urthalee F, James TN. Morphological changes in the right ventricular septomarginal trabecula (false tendon) during maturation and ageing in the dog heart. J Anat 1979; 129: 805-17.

22. Glomset DJ, Glomset ATA. A morphologic study of the cardiac conduction system in ungulates, dog, and man. Part II: the Purkinje system. Am Heart J 1940; 20: 677-701.

23. Sandusky GE, White SL. Scanning microscopy of the canine atrioventricular bundle and moderator band. Am J Vet Res 1985; 46: 249-52.

24. Krysiak K, Świeżyński K. Anatomia zwierząt. Tom II. PWN, Warszawa 1983

25. Smolich JJ, Shimada T, Canale H, Cambell GR. Developmental morphology of vascular and lymphatic capillaries in the working myocardium and Purkinje bundle of the sheep septomarginal band. Anat Rec 1990; 226: 48-56. 